\title{
Small Dense Low-Density Lipoproteins Cholesterol can Predict Incident Cardiovascular Disease in an Urban Japanese Cohort: The Suita Study
}

\author{
Hidenori Arai ${ }^{1}$, Yoshihiro Kokubo ${ }^{2}$, Makoto Watanabe ${ }^{2}$,Tatsuya Sawamura ${ }^{3}$, Yasuki Ito ${ }^{4}$, Asako Minagawa ${ }^{4}$, \\ Tomonori Okamura ${ }^{5}$ and Yoshihiro Miyamato ${ }^{2}$
}

\author{
${ }^{1}$ Department of Human Health Sciences, Kyoto University Graduate School of Medicine, Kyoto, Japan \\ ${ }^{2}$ Department of Preventive Cardiology, National Cerebral and Cardiovascular Center, Suita, Japan \\ ${ }^{3}$ Department of Vascular Physiology, National Cerebral and Cardiovascular Center, Suita, Japan \\ ${ }^{4}$ Research and Development Department, Denka Seiken, Tokyo, Japan \\ ${ }^{5}$ Department of Preventive Medicine and Public Health, School of Medicine, Keio University, Tokyo, Japan
}

\begin{abstract}
Aim: Several lines of evidence indicate that small dense low-density lipoproteins (sd-LDL) are more atherogenic than large buoyant LDL; however, few prospective studies have addressed the role of sdLDL in cardiovascular disease (CVD). We therefore examined the association between sd-LDL cholesterol (sd-LDL-C) and CVD in a Japanese cohort.

Methods: An 11.7-year prospective study was performed using a general population aged 30-79 without a history of cardiovascular disease. Direct LDL-C and sd-LDL-C were measured in samples from 2034 participants (968 men and 1066 women).

Results: During the follow-up period, there were 116 incident cases of CVD. The multivariableadjusted hazard ratios (HRs) of sd-LDL-C for CVD were calculated using a proportional hazards regression model after adjusting for age, hypertension, diabetes, use of lipid-lowering drugs, body mass index, and current smoking and alcohol drinking, and found that increasing quartiles of sdLDL-C were associated with increased risk of CVD. We also determined that age and sex-adjusted HRs per $10 \mathrm{mg} / \mathrm{dL}$ of sd-LDL-C and HRs for CVD, stroke, cerebral infarction, and coronary artery disease were 1.21 (95\% CI: 1.12-1.31), 1.17 (95\% CI: 1.05-1.30), 1.15 (95\% CI: 1.00-1.33), and 1.29 (95\% CI: 1.14-1.45), respectively.

Conclusions: It was demonstrated that sd-LDL-C was significantly associated with CVD in a Japanese population, providing evidence of sd-LDL-C as an important biomarker to predict CVD.
\end{abstract}

J Atheroscler Thromb, 2013; 20:195-203.

Key words; Cardiovascular disease, Lipoproteins, Lipids, Risk factors, Epidemiology

\section{Introduction}

The causal relationship between high levels of serum low-density lipoprotein cholesterol (LDL-C) and cardiovascular disease (CVD) has been well established in previous cohort studies ${ }^{1-5}$. Recent clinical

Address for correspondence: Hidenori Arai, Department of Human Health Sciences, Kyoto University Graduate School of Medicine, 53 Kawahara-cho, Shogoin, Sakyo-ku, Kyoto 606-8507, Japan

E-mail: harai@kuhp.kyoto-u.ac.jp

Received: June 20, 2012

Accepted for publication: September 6, 2012 trials have also indicated significant event reduction by statins in the primary and secondary prevention of $\mathrm{CVD}^{6-8)}$; therefore, LDL-C is one of the most important risk factors of CVD and many guidelines, including ours, recommend certain target LDL-C goals for risk management to prevent the development of $\mathrm{CVD}^{5}$. Although we use LDL-C as the primary target for cholesterol-lowering therapy, LDL particles are heterogeneous with respect to size and density. Compared to large, buoyant LDL, small dense LDL (sdLDL) particles exhibit a prolonged plasma residence time, increased penetration into the arterial wall, lower affinity for the LDL receptor, and increased sus- 
ceptibility to oxidation ${ }^{9)}$. Thus, sd-LDL particles possess elevated atherogenic potential. Furthermore, elevated concentrations of sd-LDL can be found in patients with type 2 diabetes, metabolic syndrome, chronic kidney disease, and familial combined hyperlipidemia ${ }^{10-14)}$, all of which have been found as highly atherogenic conditions. Although Hirano et al. showed that sd-LDL-C is significantly higher in patients with coronary artery disease (CAD) in a cross-sectional study ${ }^{14)}$, no prospective study has addressed whether sd-LDL-C can predict a risk for CVD in non-Western populations. Recently, the Québec Cardiovascular Study has shown prospectively that men with an elevated proportion of LDL with a diameter less than 25.5 $\mathrm{nm}$ had a 3.6-fold increased risk of CAD compared with men with relatively normal $\mathrm{LDL}^{15)}$, indicating the strong link of sd-LDL to CVD as a biomarker of cardiovascular disease. Due to its atherogenic properties it is useful to measure sd-LDL for risk assessment; however, a reliable routine method is lacking.

sd-LDL has been measured by ultracentrifugation $^{16)}$ or gradient gel electrophoresis ${ }^{17)}$; however, these methods are both unsuitable for routine analysis, because each requires expensive equipment, complicated techniques, and long assay times. Hirano et al. have recently developed a simple precipitation method for sd-LDL-C quantification consisting of 2 steps: removal of apolipoprotein B-containing sd-LDL-free lipoproteins by precipitation with heparin and magnesium, followed by LDL-C measurement by the homogeneous method ${ }^{18,19)}$. This assay allowed us to screen sd-LDL-C in a large cohort. Using this assay, Ai et al. recently performed a case control study using samples from the Framingham Offspring Study and found significantly higher sd-LDL-C in women with $C A D^{20)}$. Koba et al. also showed that sd-LDL-C is more powerful than LDL-C for the determination of $\mathrm{CAD}^{21)}$; however, these are cross-sectional studies and a prospective study is required to determine whether sdLDL is an independent predictor of CVD. Therefore, the aim of this study was to address the role of sdLDL-C for incident CVD in a large cohort study in Japan, the Suita study.

\section{Methods}

\section{Population}

The Suita study, a cohort study on CVD of urban residents, was established in 1989. The details of this study have been described elsewhere ${ }^{22)}$. Briefly, 6485 men and women aged 30-79 years underwent a baseline survey at the National Cerebral and Cardiovascular Center between September 1989 and March
1994, and received medical examinations every 2 years. For these participants, we set the baseline of the present study at medical examinations held between April 1994 and February 1995, since at that time serum samples were collected and stored at $-80^{\circ} \mathrm{C}$. During this period, 2,437 participants attended the medical examination and were followed until the end of 2007. Of these, 403 participants were excluded due to the following reasons: history of CAD or stroke $(n=106)$, lost to follow-up $(n=132)$, and other reasons such as missing data $(n=165)$. Data from the remaining 2,034 participants (968 men and 1,066 women) were included in the analysis. Informed consent was obtained from all participants. This cohort study was approved by the Institutional Review Board of the National Cerebral and Cardiovascular Center.

\section{Baseline Examination}

Blood samples were collected after the participants had fasted for at least 10 hours. The samples were centrifuged immediately. Blood pressure was measured in triplicate on the right arm after $5 \mathrm{~min}$ of rest by well-trained physicians using a standard mercury sphygmomanometer. The average of the second and third measurements was used for analysis. At baseline examination, subjects were classified into one of the 5 blood pressure categories based on the criteria of ESHESC 2007: optimal (SBP $<120 \mathrm{mmHg}$ and DBP $<80$ $\mathrm{mmHg}$ ), normal (SBP 120-129 mmHg or DBP 80-84 $\mathrm{mmHg}$ ), high-normal blood pressure (SBP 130-139 $\mathrm{mmHg}$ or DBP $85-89 \mathrm{mmHg}$ ), hypertension grade 1 (SBP $140-159 \mathrm{mmHg}$ or DBP $90-99 \mathrm{mmHg}$ ), or hypertension grade $\geq 2$ (SBP $\geq 160 \mathrm{mmHg}$ or DBP $\geq 100 \mathrm{mmHg}$ ). Antihypertensive drug users were classified according to their blood pressure at the baseline survey. Diabetes was defined as fasting serum glucose $\geq 7.0 \mathrm{mmol} / \mathrm{L}(126 \mathrm{mg} / \mathrm{dL})$ or current use of medications for diabetes. Body mass index (BMI) was calculated as weight (kilograms) divided by height (meters) squared. Well-trained health nurses obtained information on smoking, drinking, and medical histories.

\section{Laboratory Measurements}

Serum total cholesterol, triglyceride, and HDL cholesterol (HDL-C) were determined by standard enzymatic methods. Serum glucose was also measured. For the purposes of this study, we used archived plasma samples that had been frozen at $-80^{\circ} \mathrm{C}$ and never previously thawed for the assessment of direct LDL-C and sd-LDL-C by homogeneous methods on a Hitachi 7180 automated analyzer (Hitachi, Tokyo, Japan) ${ }^{18,19)}$. The kits used for these tests (LDL-C and sd-LDL-C) were provided by Denka Seiken (Tokyo, Japan). Assays 
for direct LDL-C and sd-LDL-C were previously calibrated and directly compared with concentrations obtained after isolation of LDL and sd-LDL by ultracentrifugation.

\section{Endpoint Determination}

As previously reported, the endpoints of the present study were (1) date of first CAD or stroke event; (2) date of death; (3) date of leaving Suita city; and (4) the end of December 2007. The first step in the survey for CAD and stroke involved checking the health status of all participants by repeated clinical visits every two years and yearly questionnaires by mail or telephone. In the second step, in-hospital medical records of participants who were suspected of having $\mathrm{CAD}$ were reviewed by registered hospital physicians or research physicians who were blinded to the baseline information. The criteria for a diagnosis of CAD included first-ever acute myocardial infarction, sudden cardiac death within $24 \mathrm{~h}$ after the onset of acute illness, or coronary artery disease followed by coronary artery bypass surgery or angioplasty. The criteria for definite and probable MI were defined according to the criteria of the MONICA (Monitoring Trends and Determinants of Cardiovascular Disease) project ${ }^{23)}$. The criteria for stroke were defined according to the US National Survey of Stroke criteria ${ }^{24)}$. Classification of patients into stroke subtypes was based on examination of computed tomography, magnetic resonance imaging, or autopsy.

\section{Statistical Analysis}

Continuous variables between groups were compared by analysis of variance and categorical variables were compared by a chi-square test. Triglyceride levels were logarithmically transformed to improve the skewed distribution. The hazard ratio (HR) for MI or stroke was calculated using a proportional hazards model adjusted for age, sex, hypertension (dichotomous variable), diabetes, HDL-C, BMI, smoking (never-smoked; ex-smoker; current smoker) and drinking (never-drank; ex-drinker; regular drinker). All confidence intervals were estimated at the 95\% level and significance was set at $p<0.05$. All statistical analyses were conducted using the SAS statistical software package (release version 8.2; SAS Institute, Cary, NC, USA).

\section{Results}

\section{Baseline Clinical Characteristics According to sd- LDL-C Quartiles}

To study the role of sd-LDL in the incidence of
CVD, we divided the cohort into quartiles according to the basal level of sd-LDL-C. Table 1 shows the clinical characteristics and cardiovascular risk factors of the study population according to the quartiles of sdLDL-C. BMI, total cholesterol, LDL-C, and triglyceride significantly increased across the sd-LDL-C quartiles both in men and women, while HDL-C decreased in both genders. A significant trend was observed across the quartiles for the severity of high blood pressure, lipid-lowering drug use, and prevalence of diabetes at baseline both in men and women; however, a significant trend for age was only found in women, not in men.

\section{Incidence of CVD According to sd-LDL-C Quartiles}

To confirm our previous study, the association between LDL-C and CAD was examined by dividing the cohort into quartiles according to the baseline LDL-C. It was found that age- and multivariableadjusted HRs for CAD were statistically significant only in men, not in women or the total cohort. The HR of the 4th quartile in men was 3.53 (95\% confidence intervals (CIs): 1.31-9.54) in an age-adjusted model and 3.56 (95\% CIs: 1.28-9.86) in a multivariable-adjusted model, consistent with our previous report $^{1)}$. We then performed analysis to examine the effect of sd-LDL-C. During the observation period, 116 cases of CVD, 53 cases of stroke, 36 cases of cerebral infarction, and 63 cases of CAD were reported. As shown in Table 2, increasing quartiles of sd-LDL$\mathrm{C}$ were significantly associated with increased risks of CVD (stroke + CAD), stroke, cerebral infarction, and $\mathrm{CAD}$ after age and multivariable adjustment. Age and sex-adjusted HRs per $10 \mathrm{mg} / \mathrm{dL}$ of sd-LDL-C for CVD, stroke, cerebral infarction, and CAD were 1.21 (95\% CI: 1.12-1.31), 1.17 (95\% CI: 1.05-1.30), 1.15 (95\% CI: 1.00-1.33), and 1.29 (95\% CI: 1.14-1.45), respectively. HRs after multivariable adjustment were almost the same. When we analyzed each gender, ageadjusted HRs per $10 \mathrm{mg} / \mathrm{dL}$ of sd-LDL-C for CVD, stroke, cerebral infarction, and CAD were significant in women, while those for CVD and CAD were significant in men. HR for CAD of the fourth quartile was almost 4 after age and multivariable adjustment in men.

After putting LDL-C into the multivariable adjusted-models (Model A), sd-LDL-C was still associated with increased risk for CVD, stroke, cerebral infarction, and CAD in the total cohort, for CVD in men, and CVD, stroke, and cerebral infarction in women. After further putting logarithmically transformed triglyceride and HDL-C variables into Model A (Model B), sd-LDL-C was still associated with 
Table 1. Baseline characteristics of cardiovascular risk factors according to small dense LDL cholesterol quartiles

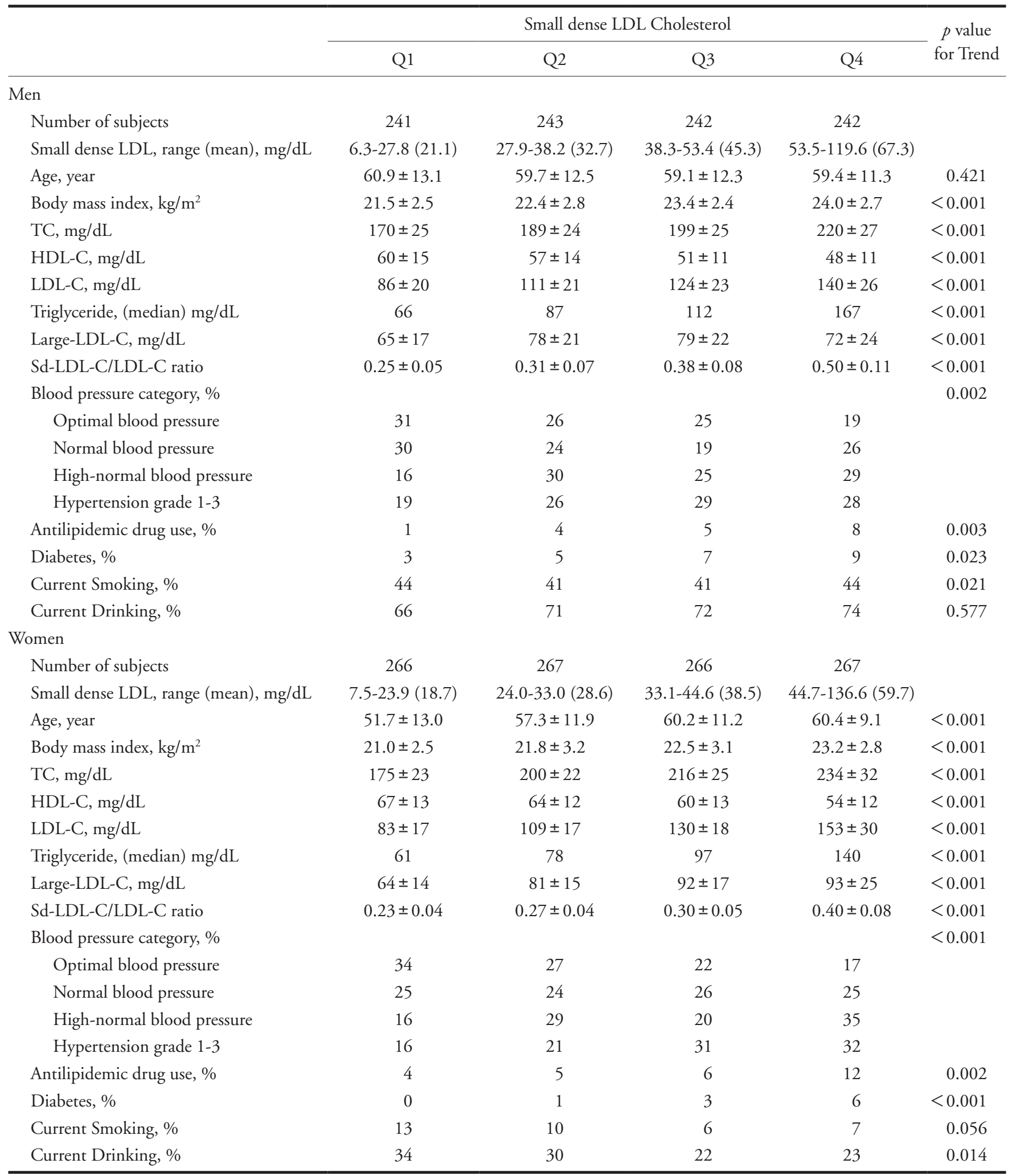

TC, total cholesterol; HDL-C, high-density lipoprotein cholesterol; LDL-C, low-density lipoprotein cholesterol. Large LDL-C, LDL-C-Sd-LDLC. Hypertension was defined as described in methods. Diabetes was defined as fasting serum glucose $\geq 7.0 \mathrm{mmol} / \mathrm{L}(126 \mathrm{mg} / \mathrm{dL})$, the use of antidiabetic agents, or both. 
Table 2. Age- and multivariable-adjusted hazard ratios and 95\% confidence intervals for the incidence of cardiovascular disease according to small dense LDL cholesterol quartiles

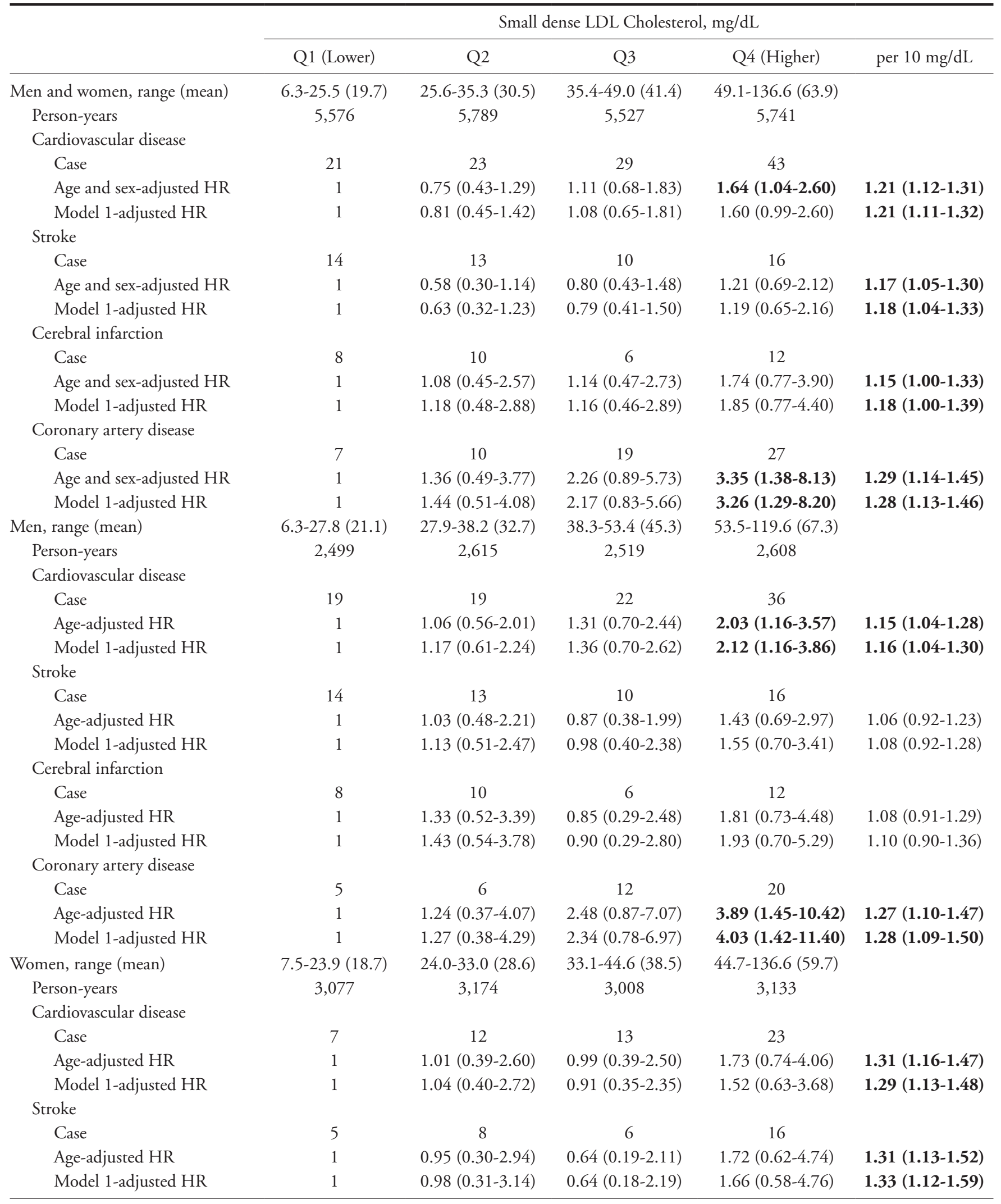




\begin{tabular}{|c|c|c|c|c|c|}
\hline & \multicolumn{5}{|c|}{ Small dense LDL Cholesterol, mg/dL } \\
\hline \multicolumn{6}{|l|}{ Cerebral infarction } \\
\hline Case & 0 & 5 & 4 & 7 & \\
\hline Age-adjusted HR & 1 & - & - & - & $1.31(1.05-1.63)$ \\
\hline Model 1-adjusted HR & 1 & - & - & - & $1.37(1.05-1.80)$ \\
\hline Case & 2 & 4 & 7 & 7 & \\
\hline Age-adjusted HR & 1 & $1.22(0.22-7.76)$ & $1.90(0.39-9.24)$ & $1.84(0.38-8.91)$ & $1.32(1.08-1.61)$ \\
\hline Model 1-adjusted HR & 1 & $1.27(0.22-7.33)$ & $1.83(0.35-9.45)$ & $1.54(0.30-7.83)$ & $1.23(0.99-1.53)$ \\
\hline
\end{tabular}

Model 1: adjusted for age, (sex), body mass index, smoking, drinking, blood pressure category (optimal, normal, and high-normal blood pressure, hypertension grade 1 and $2+3$ ), diabetes, and lipid-lowering drug user Bold numbers: statistically significant

increased risks of CVD and stroke in the total cohort and in women, but not in men (Table 3).

\section{Discussion}

This study clearly indicates an increased risk of CVD, stroke, cerebral infarction, and CAD attributed to elevated sd-LDL-C concentrations in a Japanese population without a previous history of CVD. We also showed that HR was significant after multivariable adjustment and by analysis including LDL-C, log-transformed triglyceride, and HDL-C in the same model. Thus, sd-LDL-C measurement with the new test is promising as a new biomarker to predict the risk of CVD.

In addition to traditional risk factors for CVD, such as hypertension, diabetes, and dyslipidemia, other biomarkers are required to better define the risk and refine therapeutic decisions. There is scientific evidence that sd-LDL particles are highly atherogenic and can be a biomarker of CVD ${ }^{15,20,21)}$. Our data provide additional evidence to show the role of sd-LDL$\mathrm{C}$ as a CVD risk in the general population. Furthermore, measuring sd-LDL-C with this test has an advantage because it is more user-friendly and more applicable than specialized tests such as gradient gel electrophoresis, nuclear magnetic resonance, and gradient ultracentrifugation.

Until now, there have been no target goals of sdLDL-C to prevent CAD. In this study the HR of the 4th quartile was statistically significant, suggesting that the cutoff of sd-LDL-C is approximately $50 \mathrm{mg} /$ $\mathrm{dL}$, although significance was not obtained in women probably due to the low event rate; therefore, a larger study should be performed to define an appropriate cutoff for sd-LDL-C. Because statins, fibrates, and ezetimibe have been shown to reduce the amount of sd-LDL ${ }^{25-28)}$, a randomized control study is required to address whether lowering sd-LDL-C to a certain goal by these drugs can prevent the development of CAD.

In this study we found that sd-LDL-C was significantly associated with traditional risk factors, such as hypertension and diabetes. BMI and the prevalence of diabetes increased and HDL-C decreased across the sd-LDL-C quartiles, and more hypertensive subjects were found in second to fourth quartiles than in the first quartile in both genders. We also found that ageadjusted partial correlation coefficients between sdLDL-C and BMI, log-transformed triglyceride, LDL$\mathrm{C}$, and HDL-C (Pearson) were 0.305, 0.636, 0.554, and $-0.346(p<0.0001)$, respectively. Thus these data suggest that increased concentrations of sd-LDL-C may be associated with metabolic disorders and that lifestyle modification, such as exercise and weight control, would be effective to reduce sd-LDL in patients with diabetes and metabolic syndrome. Furthermore, we should address whether sd-LDL-C can be used to identify a very high-risk patient with type 2 diabetes, metabolic syndrome, and other metabolic disorders. In contrast to the association with metabolic disorders, an age-related change in sd-LDL-C was found only in women, consistent with the trend of increased atherogenic dyslipidemia in postmenopausal women. Ai et al. also found that postmenopausal women had higher levels of sd-LDL-C than premenopausal women in the Framingham Offspring Study ${ }^{20)}$.

In addition to type 2 diabetes and metabolic syn- 
Table 3. Relationship between major lipid variables and cardiovascular disease

\begin{tabular}{|c|c|c|c|c|}
\hline & $\begin{array}{l}\text { Cardiovascular } \\
\text { disase }\end{array}$ & Stroke & $\begin{array}{l}\text { Cerebral } \\
\text { infarction }\end{array}$ & $\begin{array}{l}\text { Coronary artery } \\
\text { disease }\end{array}$ \\
\hline \multicolumn{5}{|l|}{ Men and women } \\
\hline Age and sex-adjusted & $1.21(1.12-1.31)$ & $1.17(1.05-1.30)$ & $1.15(1.00-1.33)$ & $1.29(1.14-1.45)$ \\
\hline $\begin{array}{l}\text { Multivariable-adjusted Sd-LDL-C/10 mg/dL } \\
\text { Model A }\end{array}$ & $1.21(1.11-1.32)$ & $1.18(1.04-1.33)$ & $1.18(1.00-1.39)$ & $1.28(1.13-1.46)$ \\
\hline Sd-LDL-C/10 mg/dL & $1.26(1.11-1.43)$ & $1.26(1.06-1.50)$ & $1.29(1.02-1.62)$ & $1.29(1.07-1.55)$ \\
\hline $\mathrm{LDL}-\mathrm{C} / 10 \mathrm{mg} / \mathrm{dL}$ & $0.96(0.89-1.04)$ & $0.94(0.85-1.04)$ & $0.93(0.81-1.06)$ & $0.99(0.88-1.11)$ \\
\hline \multicolumn{5}{|l|}{ Model B } \\
\hline Sd-LDL-C/10 mg/dL & $1.20(1.01-1.42)$ & $1.35(1.07-1.71)$ & $1.31(0.96-1.78)$ & $1.05(0.81-1.36)$ \\
\hline $\mathrm{LDL}-\mathrm{C} / 10 \mathrm{mg} / \mathrm{dL}$ & $0.98(0.90-1.06)$ & $0.93(0.83-1.03)$ & $0.92(0.80-1.07)$ & $1.05(0.93-1.19)$ \\
\hline ln_TG & $1.15(0.71-1.86)$ & $0.76(0.40-1.46)$ & $0.86(0.37-1.96)$ & $1.82(0.87-3.81)$ \\
\hline $\mathrm{HDL}-\mathrm{C} / 10 \mathrm{mg} / \mathrm{dL}$ & $0.94(0.81-1.08)$ & $1.00(0.84-1.20)$ & $0.93(0.73-1.18)$ & $0.80(0.61-1.04)$ \\
\hline \multicolumn{5}{|l|}{ Men } \\
\hline Age-adjusted & $1.15(1.04-1.28)$ & $1.06(0.92-1.23)$ & $1.08(0.91-1.29)$ & $1.27(1.10-1.47)$ \\
\hline \multicolumn{5}{|l|}{ Model A } \\
\hline Sd-LDL-C/10 mg/dL & $1.17(1.00-1.38)$ & $1.17(0.92-1.48)$ & $1.20(0.90-1.60)$ & $1.18(0.94-1.48)$ \\
\hline LDL-C/10 mg/dL & $0.99(0.89-1.09)$ & $0.94(0.82-1.08)$ & $0.93(0.79-1.09)$ & $1.07(0.93-1.24)$ \\
\hline \multicolumn{5}{|l|}{ Model B } \\
\hline Sd-LDL-C/10 mg/dL & $1.10(0.88-1.38)$ & $1.28(0.92-1.77)$ & $1.28(0.87-1.90)$ & $0.96(0.70-1.31)$ \\
\hline $\mathrm{LDL}-\mathrm{C} / 10 \mathrm{mg} / \mathrm{dL}$ & $1.01(0.90-1.13)$ & $0.92(0.78-1.07)$ & $0.91(0.76-1.10)$ & $1.14(0.97-1.33)$ \\
\hline ln_TG & $1.23(0.66-2.26)$ & $0.75(0.32-1.76)$ & $0.86(0.31-2.38)$ & $1.87(0.75-4.62)$ \\
\hline HDL-C/10 mg/dL & $0.96(0.80-1.14)$ & $1.05(0.85-1.28)$ & $1.08(0.94-1.40)$ & $0.72(0.50-1.03)$ \\
\hline \multicolumn{5}{|l|}{ Women } \\
\hline Age-adjusted & $1.31(1.16-1.47)$ & $1.31(1.13-1.52)$ & $1.31(1.05-1.63)$ & $1.32(1.08-1.61)$ \\
\hline $\begin{array}{l}\text { Multivariable-adjusted Sd-LDL-C/10 mg/dL } \\
\text { Model A }\end{array}$ & $1.29(1.13-1.48)$ & $1.33(1.12-1.59)$ & $1.37(1.05-1.80)$ & $1.23(0.99-1.53)$ \\
\hline Sd-LDL-C/10 mg/dL & $1.44(1.17-1.77)$ & $1.48(1.13-1.94)$ & $1.62(1.08-2.43)$ & $1.33(0.94-1.89)$ \\
\hline LDL-C/10 mg/dL & $0.92(0.81-1.04)$ & $0.92(0.79-1.08)$ & $0.88(0.69-1.11)$ & $0.94(0.75-1.16)$ \\
\hline \multicolumn{5}{|l|}{ Model B } \\
\hline Sd-LDL-C/10 mg/dL & $1.35(1.03-1.77)$ & $1.47(1.04-2.08)$ & $1.33(0.78-2.29)$ & $1.12(0.70-1.79)$ \\
\hline LDL-C/10 mg/dL & $0.93(0.81-1.07)$ & $0.92(0.78-1.09)$ & $0.92(0.72-1.19)$ & $0.98(0.78-1.24)$ \\
\hline ln_TG & $1.19(0.53-2.69)$ & $0.91(0.31-2.68)$ & $0.86(0.17-4.25)$ & $1.84(0.47-7.15)$ \\
\hline HDL-C/10 mg/dL & $0.92(0.72-1.19)$ & $0.92(0.67-1.26)$ & $0.56(0.31-1.00)$ & $0.92(0.60-1.41)$ \\
\hline
\end{tabular}

Multivariable adjusted for age, sex, body mass index, smoking, drinking, blood pressure category (optimal, normal, and high-normal bloodpressure, hypertension grade 1 and $2+3$ ), diabetes, and antilipidemic drug user

Model A: sd-LDL-C per $10 \mathrm{mg} / \mathrm{dL}$ and LDL-C per $10 \mathrm{mg} / \mathrm{dL}$ in the same model

Model B: sd-LDL-C per $10 \mathrm{mg} / \mathrm{dL}$, LDL-C per $10 \mathrm{mg} / \mathrm{dL}, \ln (\mathrm{TG})$, and HDL-C per $10 \mathrm{mg} / \mathrm{dL}$ in the same model

Sd-LDL-C, small dense LDL cholesterol; $\ln \_$TG, logarithmical transformed TG

Bold numbers: statistically significant

drome, sd-LDL-C is increased in familial combined hyperlipidemia and postprandial hyperlipidemia ${ }^{29,30)}$. Hirano et al. demonstrated that sd-LDL-C determined by this simple precipitation method is useful for screening familial combined hyperlipidemia in large populations ${ }^{13)}$. Because the prevalence of familial combined hyperlipidemia is high in the general population and the increase of sd-LDL particles as well as large VLDL particles is a characteristic feature of familial combined hyperlipidemia, this assay would be quite useful for its diagnosis. Although sd-LDL is decreased by lipid-lowering drugs, such as statins and fibrates, the effect of adequate combination therapy on sd-LDL-C has not yet been confirmed; therefore, this assay would be also useful in determining the therapeutic strategy for patients with a high serum level of sd-LDL-C.

There are some limitations in our study. First, we 
used plasma stored at $-80{ }^{\circ} \mathrm{C}$, and there is no guarantee that we would have obtained the same results if we had used fresh serum; however, our results are consistent with those reported by Hirano et al., who measured sd-LDL-C in a Japanese general population with the same method, and comparison studies performed in Japan indicate virtually identical results with the use of fresh vs. frozen plasma for sd-LDL-C ${ }^{13)}$. Second, the single measurement of sd-LDL-C at the baseline survey and the fact we did not evaluate the longitudinal trend for each risk factor including lipid-lowering agents may have caused us to underestimate the relationship between these conditions and CAD due to regression dilution bias, although we statistically adjusted for the use of lipid-lowering agents at the baseline survey. Third, serum LDL-C was measured by the direct homogeneous assay, which failed to meet the National Cholesterol Education Program total error goals for diseased individuals, although it met these goals in non-diseased individuals ${ }^{31)}$. However, the present study is a cohort study of communitydwelling citizens without a history of CVD. Furthermore, the serum levels of LDL-C determined by direct homogeneous assay are almost consistent with those calculated by the Friedewald formula in a large Japanese cohort.

\section{Conclusions}

In this large urban cohort study conducted in Japan, we demonstrated that sd-LDL-C is significantly associated with the development of CVD, providing evidence of sd-LDL-C as an important biomarker to predict CVD. A large intervention study is required to determine the appropriate target level of sd-LDL-C.

\section{Sources of Funding}

The present study was supported by the Intramural Research Fund of the National Cerebral and Cardiovascular Center (22-4- 5), and also supported by grants-in-aid from the Ministry of Health, Labor and Welfare (H22-Seishu-005).

\section{Disclosures}

Dr. Arai has received an unrestricted grant from Denka Seiken. Drs Ito and Minagawa are employees of Denka Seiken. The other authors have no conflicts to declare.

\section{References}

1) Okamura T, Kokubo Y, Watanabe M, Higashiyama A, Miyamoto Y, Yoshimasa Y, Okayama A: Low-density lipoprotein cholesterol and non-high-density lipoprotein cholesterol and the incidence of cardiovascular disease in an urban Japanese cohort study: The Suita study. Atherosclerosis, 2009; 203: 587-592

2) Law MR, Wald NJ: An ecological study of serum cholesterol and ischaemic heart disease between 1950 and 1990. Eur J Clin Nutr, 1994; 48: 305-325

3) Law MR, Wald NJ, Rudnicka AR: Quantifying effect of statins on low density lipoprotein cholesterol, ischaemic heart disease, and stroke: systematic review and metaanalysis. BMJ, 2003; 326: 1423

4) Prospective Studies C, Lewington S, Whitlock G, Clarke R, Sherliker P, Emberson J, Halsey J, Qizilbash N, Peto R, Collins R: Blood cholesterol and vascular mortality by age, sex, and blood pressure: a meta-analysis of individual data from 61 prospective studies with 55,000 vascular deaths. Lancet, 2007; 370: 1829-1839

5) Teramoto T, Sasaki J, Ueshima H, Egusa G, Kinoshita M, Shimamoto K, Daida H, Biro S, Hirobe K, Funahashi T, Yokote K, Yokode M: Executive summary of Japan Atherosclerosis Society (JAS) guideline for diagnosis and prevention of atherosclerotic cardiovascular diseases for Japanese. J Atheroscler Thromb, 2007; 14: 45-50

6) Nakamura H, Arakawa K, Itakura H, Kitabatake A, Goto Y, Toyota T, Nakaya N, Nishimoto S, Muranaka M, Yamamoto A, Mizuno K, Ohashi Y: Primary prevention of cardiovascular disease with pravastatin in Japan (MEGA Study): a prospective randomised controlled trial. Lancet, 2006; 368: 1155-1163

7) Ridker PM, Danielson E, Fonseca FA, Genest J, Gotto AM Jr, Kastelein JJ, Koenig W, Libby P, Lorenzatti AJ, MacFadyen JG, Nordestgaard BG, Shepherd J, Willerson JT, Glynn RJ: Rosuvastatin to prevent vascular events in men and women with elevated C-reactive protein. N Engl J Med, 2008; 359: 2195-2207

8) Baigent C, Keech A, Kearney PM, Blackwell L, Buck G, Pollicino C, Kirby A, Sourjina T, Peto R, Collins R, Simes R: Efficacy and safety of cholesterol-lowering treatment: prospective meta-analysis of data from 90,056 participants in 14 randomised trials of statins. Lancet, 2005; 366: $1267-1278$

9) Berneis KK, Krauss RM: Metabolic origins and clinical significance of LDL heterogeneity. J Lipid Res, 2002; 43: 1363-1379

10) Reaven GM, Chen YD, Jeppesen J, Maheux P, Krauss RM: Insulin resistance and hyperinsulinemia in individuals with small, dense low density lipoprotein particles. J Clin Invest, 1993; 92: 141-146

11) Kathiresan S, Otvos JD, Sullivan LM, Keyes MJ, Schaefer EJ, Wilson PW, D'Agostino RB, Vasan RS, Robins SJ: Increased small low-density lipoprotein particle number: a prominent feature of the metabolic syndrome in the Framingham Heart Study. Circulation, 2006; 113: 20-29

12) Deighan CJ, Caslake MJ, McConnell M, Boulton-Jones JM, Packard CJ: Atherogenic lipoprotein phenotype in end-stage renal failure: origin and extent of small dense 
low-density lipoprotein formation. Am J Kidney Dis, 2000; 35: 852-862

13) Hirano T, Nohtomi K, Sato Y, Kamata K, Ito Y: Small dense LDL-cholesterol determined by a simple precipitation assay for screening familial combined hyperlipidemia. Atherosclerosis, 2009; 205: 603-607

14) Hirano T, Ito $Y$, Koba $S$, Toyoda M, Ikejiri A, Saegusa $H$, Yamazaki J, Yoshino G: Clinical significance of small dense low-density lipoprotein cholesterol levels determined by the simple precipitation method. Arterioscler Thromb Vasc Biol, 2004; 24: 558-563

15) St-Pierre AC, Cantin B, Dagenais GR, Mauriege P, Bernard PM, Despres JP, Lamarche B: Low-density lipoprotein subfractions and the long-term risk of ischemic heart disease in men: 13-year follow-up data from the Quebec Cardiovascular Study. Arterioscler Thromb Vasc Biol, 2005; 25: 553-559

16) Swinkels DW, Hak-Lemmers HL, Demacker PN: Single spin density gradient ultracentrifugation method for the detection and isolation of light and heavy low density lipoprotein subfractions. J Lipid Res, 1987; 28: 1233-1239

17) Nichols AV, Krauss RM, Musliner TA: Nondenaturing polyacrylamide gradient gel electrophoresis. Methods Enzymol, 1986; 128: 417-431

18) Hirano T, Ito Y, Saegusa H, Yoshino G: A novel and simple method for quantification of small, dense LDL. J Lipid Res, 2003; 44: 2193-2201

19) Ito Y, Fujimura M, Ohta M, Hirano T: Development of a homogeneous assay for measurement of small dense LDL cholesterol. Clin Chem, 2011; 57: 57-65

20) Ai M, Otokozawa S, Asztalos BF, Ito Y, Nakajima K, White CC, Cupples LA, Wilson PW, Schaefer EJ: Small dense LDL cholesterol and coronary heart disease: results from the Framingham Offspring Study. Clin Chem, 2010; 56: 967-976

21) Koba S, Yokota Y, Hirano T, Ito Y, Ban Y, Tsunoda F, Sato T, Shoji M, Suzuki H, Geshi E, Kobayashi Y, Katagiri T: Small LDL-cholesterol is superior to LDL-cholesterol for determining severe coronary atherosclerosis. J Atheroscler Thromb, 2008; 15: 250-260

22) Mannami T, Baba S, Ogata J: Strong and significant relationships between aggregation of major coronary risk factors and the acceleration of carotid atherosclerosis in the general population of a Japanese city: the Suita Study. Arch Intern Med, 2000: 160: 2297-2303

23) Tunstall-Pedoe H, Vanuzzo D, Hobbs M, Mahonen M, Cepaitis Z, Kuulasmaa K, Keil U: Estimation of contribu- tion of changes in coronary care to improving survival, event rates, and coronary heart disease mortality across the WHO MONICA Project populations. Lancet, 2000; 355: 688-700

24) Walker AE, Robins M, Weinfeld FD: The National Survey of Stroke. Clinical findings. Stroke, 1981; 12: I13-44

25) Mudd JO, Borlaug BA, Johnston PV, Kral BG, Rouf R, Blumenthal RS, Kwiterovich PO Jr: Beyond low-density lipoprotein cholesterol: defining the role of low-density lipoprotein heterogeneity in coronary artery disease. J Am Coll Cardiol, 2007; 50: 1735-1741

26) Otvos JD, Collins D, Freedman DS, Shalaurova I, Schaefer EJ, McNamara JR, Bloomfield HE, Robins SJ: Lowdensity lipoprotein and high-density lipoprotein particle subclasses predict coronary events and are favorably changed by gemfibrozil therapy in the Veterans Affairs High-Density Lipoprotein Intervention Trial. Circulation, 2006; 113: 1556-1563

27) Vakkilainen J, Steiner G, Ansquer JC, Aubin F, Rattier S, Foucher C, Hamsten A, Taskinen MR, Group, D: Relationships between low-density lipoprotein particle size, plasma lipoproteins, and progression of coronary artery disease: the Diabetes Atherosclerosis Intervention Study (DAIS). Circulation, 2003; 107: 1733-1737

28) Rizzo M, Rini GB, Spinas GA, Berneis K: The effects of ezetimibe on LDL-cholesterol: quantitative or qualitative changes? Atherosclerosis, 2009; 204: 330-333

29) Veerkamp MJ, de Graaf J, Bredie SJ, Hendriks JC, Demacker PN, Stalenhoef AF: Diagnosis of familial combined hyperlipidemia based on lipid phenotype expression in 32 families: results of a 5-year follow-up study. Arterioscler Thromb Vasc Biol, 2002; 22: 274-282

30) Tsunoda F, Koba S, Hirano T, Ban Y, Iso Y, Suzuki H, Geshi E, Katagiri, T: Association between small dense low-density lipoprotein and postprandial accumulation of triglyceride-rich remnant-like particles in normotriglyceridemic patients with myocardial infarction. Circ J, 2004; 68: 1165-1172

31) Miller WG, Myers GL, Sakurabayashi I, Bachmann LM, Caudill SP, Dziekonski A, Edwards S, Kimberly MM, Korzun WJ, Leary ET, Nakajima K, Nakamura M, Nilsson G, Shamburek RD, Vetrovec GW, Warnick GR, Remaley AT: Seven direct methods for measuring HDL and LDL cholesterol compared with ultracentrifugation reference measurement procedures. Clin Chem, 2010; 56 : 977-986 